Revue d'histoire de l'Amérique française

AR REVUE D.HISTOIRE DE L'AMÉRIQUE FRANÇAISE

\title{
Ludger Duvernay et les débuts de la presse périodique aux Trois-Rivières
}

Yves Tessier

Volume 18, numéro 3, décembre 1964

URI : https://id.erudit.org/iderudit/302390ar

DOI : https://doi.org/10.7202/302390ar

Aller au sommaire du numéro

Éditeur(s)

Institut d'histoire de l'Amérique française

ISSN

0035-2357 (imprimé)

1492-1383 (numérique)

Découvrir la revue

Citer cet article

Tessier, Y. (1964). Ludger Duvernay et les débuts de la presse périodique aux Trois-Rivières. Revue d'histoire de l'Amérique française, 18(3), 387-404.

https://doi.org/10.7202/302390ar d'utilisation que vous pouvez consulter en ligne.

https://apropos.erudit.org/fr/usagers/politique-dutilisation/ 


\title{
LUDGER DUVERNAY ET LES DÉBUTS DE LA PRESSE PÉRIODIQUE AUX TROIS-RIVIÈRES*
}

\author{
I NTRODUCTION
}

Les journaux constituent une source importante de renseignements pour le chercheur qui se consacre à la connaissance du passé humain. Mais "il n'existe malheureusement pas une histoire des périodiques du Québec, et il est impossible de songer à en rédiger une d'ici plusieurs années, c'est-à-dire tant qu'un certain nombre de monographies ne pourront pas servir de base à une synthèse" ${ }^{1}$. La présente étude veut mettre en lumière quelques traits biographiques mal connus du journalisteimprimeur Ludger Duvernay, et par la suite, faire connaître les premières manifestations de la presse trifluvienne.

La vie de Ludger Duvernay n'a pas encore fait l'objet d'une étude valable. La plaquette que lui consacre Roger Parent ${ }^{2}$, bien que couvrant l'ensemble de la vie du personnage, manque d'abondance dans l'information et de profondeur dans les détails. On connaît Duvernay surtout parce qu'il a fondé la Société Saint-Jean-Baptiste en 1834. Sulte à ce titre lui consacre quelques pages ${ }^{3}$.

Même si le rôle de Duvernay parmi les Patriotes est loin d'être déterminé d'une manière satisfaisante, ses débuts dans le journalisme et l'imprimerie demeurent encore plus obscurs. Le personnage étudié a fait son apprentissage à Montréal, puis

\footnotetext{
Thèse soutenue en mai 1964, à l'Institut d'histoire de l'Université Laval (Québec).

1 Jean-Charles Bonenfant, Inventaire des sources, les journaux, dans Recherches sociographiques, juillet-décembre 1961: 495.

2 Roger D. Parent, Duvernay le Magnifique (Montréal, 1943).

3 Benjamin Sulte, La Société Saint-Jean-Baptiste 1636-1852.
} 
est venu aux Trois-Rivières fonder quatre périodiques. La connaissance de son rôle social dans cette ville a besoin d'être approfondie.

Aucun des quatre premiers journaux publiés aux TroisRivières n'a fait l'objet d'un travail monographique, si ce n'est les remarques très intéressantes de l'abbé Vallée ${ }^{4}$. C'est pourquoi de nombreuses imprécisions entourent la connaissance de ces feuilles, comme les cing du genre, relevées dans ce court extrait:"Others papers of the period were La Gazette of TroisRivieres, 1820; L'Ami de la Religion of the same year; $L a$ Constitution, 1823; and L'Argus of 1825" '5. Déterminer d'une façon précise l'existence des faits constitue déjà un travail d'envergure, nécessaire de toute évidence.

Le premier chapitre de notre travail tentera de bien isoler les principaux éléments biographiques de Ludger Duvernay, de sa naissance à 1827. Nous essaierons de connaître son apprentissage, de nous enquérir de son métier d'imprimeur aux TroisRivières, de déterminer le rôle social qu'il a pu exercer. Puis, au cours de notre deuxième chapitre, nous passerons à l'étude de chacun des journaux fondés par Duvernay dans cette ville, sans toutefois tomber dans une analyse exhaustive de leur contenu.

\section{Chapitre I}

Ludger Duvernay appartient à une importante famille établie en Nouvelle-France dès la première moitié du dix-septième siècle. Christophe Crevier arrive aux Trois-Rivières en $1639^{6}$, et se fait concéder en 1654 une importante partie du fief SaintChristophe ${ }^{7}$. La descendance de cet ancêtre sera désormais connue sous le nom de Duvernay. Ce changement s'effectue dès avril 1675, alors que le fils de Christophe, Jean-Baptiste, signe

\footnotetext{
${ }^{4}$ Henri Vallée, Les journaux trifluviens de 1817 à 1933.

5 George F. Wright, Journalism, dans The Storied Province of Quebec Past and Present, 589.

${ }^{6}$ Henri Vallée, Journaux et journalistes d'autrefois, dans Le Nouvelliste, 10 juillet 1948: 11 .

7 Benjamin Sulte, La Saint-Jean-Baptiste 1636-1852, 92.
} 
le nom Duvernay au lieu de Crevier, au bas d'un acte passé au Cap-de-la-Madeleine ${ }^{8}$. Le fils de ce dernier, Pierre, s'établit à Verchères; un de ses enfants, Jacques, occupe la charge de notaire royal dans cette région ${ }^{9}$. Un fils de Jacques, nommé Joseph-Marie, épouse en secondes noces, à Varennes, MarieAnne-Julie Rocbert de Lamorandière, le 17 avril 1792, et de leur union naît Joseph-Ludger, en 1799.

Se rattachant à la sixième génération canadienne-française de la famille Crevier-Duvernay, Joseph-Ludger est le sixième enfant né du second mariage de son père. Au moment de la naissance de Ludger, le 22 janvier 1799, seules ses sœurs MarieHortense et Julie vivent encore. Tous les autres frères et sœurs, au nombre de douze, n'ont vécu que quelques mois.

$\mathrm{Au}$ début de l'an 1799, le vicaire Labroquerie de Verchères écrit dans le registre paroissial l'acte suivant:

L'an mil sept cent quatre vingt dix neuf le Vingt deux de Janvier par moi prêtre soussigné vicaire a été baptisé Joseph Ludger né de ce matin du légitime mariage du sieur joseph crevier Duvernay maître menuisier du lieu et de dame Marie Anne julie Lamorandière. Le parrein a été le sieur François Crevier Duvernay cousin de l'enfant, la marreine Dame Adélaide Lamorandière Marchand qui ont tous signé avec nous comme cy après ainsi que le père ${ }^{10}$.

Ludger Duvernay passe son enfance dans le village natal, sans doute comme aide dans la boutique paternelle. Que deviendra le cadet de la famille? Le 3 juin 1813 dans le Spectateur de Montréal paraît l'offre d'emploi suivante: "On a besoin comme APPRENTI dans cette Imprimerie, d'une Jenne Garçon bien élevé et d'honnête famille. Il faut qu'il sache lire et écrire la langue Françoise" ${ }^{11}$. A l'âge de 14 ans, Ludger n'est-il pas ce

8 Ibid., 99.

9 D'après T. de Montigny, Histoire du droit canadien (Montréal, 1869), 586, rapporté par Sulte, op. cit., 103.

10 Cité par Parent, Duvernay le Magnifique, 7.

11 Le Spectateur: 7, col. 3. 
jeune homme sérieux que recherche l'imprimeur montréalais pour manipuler les formes typographiques et à l'occasion corriger les fautes contre la grammaire? Duvernay tente sa chance et, peu de temps plus tard, nous le retrouvons dans l'atelier de Charles-Bernard Pasteur en train de s'initier à la technique de l'imprimerie.

Cet imprimeur a tôt fait de remarquer les talents prometteurs du jeune homme de la campagne. L'impression première que donne le jeune apprenti justifie de jour en jour le choix de ce candidat. Et la bonne nouvelle ne tarde pas à atteindre la famille. La mère de Ludger lui écrit, peu de temps après son entrée à l'atelier d'impression du Spectateur: "C'est avec toute la satisfaction possible lorsque j'ai entendue M. Côté faire ton éloge dans les termes les plus flateurs il m'a dit que tu raisonnais non pas comme un perroquet mais comme un homme sensé ${ }^{12}$." La comparaison imagée revient tout à l'avantage du jeune Ludger.

Le maître-menuisier de Verchères, écrit Madame Duvernay, se réjouit du succès que remporte son fils ${ }^{13}$. Mais elle ajoute: “j'espère qu'un jour mon cher fils que par ta bonne conduite être dédommagé des peines que l'on se donne pour toi" ${ }^{14}$. La famille semble éprouver beaucoup de difficultés à supporter le séjour de Ludger à Montréal durant son apprentissage. Car l'état délabré de la fortune familiale permet difficilement de venir en aide au jeune apprenti, comme le fait remarquer Madame Duvernay: "je suis bien puni de n'avoir pas plus de fortune pour donner à mes enfans il me faut que je me borne à n'offrir que mon amitié" ${ }^{15}$. De plus, il ne faut pas perdre de vue la crise agricole qui sévit à ce moment et afflige durement les habitants: "l'été est bien dure les vivres sont d'un prix exhorbitant" ${ }^{16}$.

12 Madame Duvernay à son fils, 1814, APQ, Papiers Duvernay, pièce

2. Désormais, les Papiers Duvernay seront cités sous le sigle PD.

${ }^{13}$ Loc. cit.

14 Loc. cit.

15 Madame Duvernay à son fils, sans date, $\mathrm{PD}$, pièce $4 \mathrm{a}$.

16 Loc. cit. 
Un jeune homme aussi "sensê" peut-il demeurer longtemps apprenti dans l'atelier qui lui sert d'école? Il est tout normal que Duvernay songe à entrer au service de son maître. Il réalise cette aspiration en $\mathbf{1 8 1 5}$ avec l'appui de son précepteur, mais non sans avoir vaincu l'opposition paternelle. Louis-Généreux Labadie, maître d'école de Verchères, connaît bien le jeune Ludger. C'est la personne toute désignée pour appuyer la demande de l'apprenti: “j'ai écrit à $\mathrm{Mr}$ Pasteur comme un de vos meilleurs pilliers et je vous assure de votre place avec une augmentation que vous n'aviez point, il ne dépend qu'à vous d'y entrer" ${ }^{17}$. Cette promotion éventuelle dans les métiers de l'imprimerie suscite la réticence du père de Duvernay. Mais le précepteur incite fortement l'apprenti à vaincre l'opposition paternelle: "Approchez donc votre papa et forcez le d'embrasser vos inclinations - qui sont aussi les miennes et de votre tendre Mère qui voudrait votre bien [.] ne perdez plus de temps, dite à votre père que je le prie de m'accorder cette grace - c'est pour vous seul que je travaille - et pour lui la satisfaction d'avoir un fils imprimeur et instruit ${ }^{18}$." L'augmentation de salaire offerte par M. Pasteur témoigne de son désir de prendre Ludger Duvernay à son service. Cette promotion n'a pu tarder. Labadie semble s'intéresser de plus près à la publication du Spectateur canadien puisqu'il en devient l'agent à Verchères, à partir du 5 juin 1815. Sans doute il ne veut manquer de lire aucun numéro de ce papier-nouvelles publié maintenant avec la collaboration de Duvernay.

La conduite du jeune homme à Montréal laisse déjà entrevoir les traits de la personnalité qui se dessine. La famille Pasteur, chez qui demeure l'apprenti-imprimeur, noue d'étroits liens d'amitié avec les Duvernay de Verchères. Les témoignages d'appréciation que la fille du maître-imprimeur, Cécile, fait parvenir aux parents du pensionnaire révèlent le caractère sérieux et à la fois très affable du jeune homme. Duvernay se consacre uniquement à son travail d'une façon très intéressée, pièce 4

${ }^{17}$ Louis-Généreux Labadie à Ludger Duvernay, 1 février 1815, PD, ${ }_{18}^{4}$ Loc. cit. 
comme le fait remarquer Cécile Pasteur: "vous ne serez pas fâchée que je vous dise quelque chose de lui [Ludger] puisque c'est du bien, il ne sort presque jamais sans avoir affaire" ${ }^{19}$.

Le sérieux avec lequel il s'acquitte de son travail n'exclue pas un air jovial qui égaie la famille. Car, au dire de Cécile, Ludger "est toujours à la maison avec nous il est toujours gai et de bonne humeur. Maman s'attache à lui tous les jours davantage elle ne lui a jamais fait tant d'amitié que depuis quelques tems" ${ }^{20}$. Lorsque Duvernay s'absente, il apporte avec lui la gaieté coutumière de la maison. C'est pourquoi son retour est attendu avec impatience. Cécile commente ainsi l'arrivée de Ludger, parti en promenade à Verchères: "Le retour de Ludger a ramener la gaieté dans la maison car tous les jeux était arrêter depuis son départ; quoi qu'il est rester moins longtemps que de coutume chaqu'un se plaignait de son absence ${ }^{21}$." La personnalité attachante du jeune Duvernay laisse déjà sa trace sur son entourage.

Sur le plan de son travail, Duvernay jouit-il d'une estime aussi grande? Il n'est pas facile de préciser la place qu'il occupe dans l'atelier de Pasteur. Cependant on peut croire que l'imprimeur montréalais a tôt fait de confier à Duvernay la tâche importante de l'assister directement dans son travail. $\mathrm{Du}$ moins, en l'absence de Pasteur parti à Québec, c'est à Duvernay qu'on s'adresse pour régler des questions d'impression. Ainsi un lecteur de l'Assomption écrit à M. Duvernay, "junior", chez M. C. B. Pasteur: "Comme le jeune Mr Bazil Mignault m'a annoncé que Mr Pasteur Etait à Québec et qu'il devait être de retour qu'à la fin de cette semaine ou au Commencement de l'autre je prendrai la liberthé de vous informer que la lettre que je lui ai adressé dernièrement était pour le prier de ne pas donner place dans le Spectateur à la Communication que lui a adressé l'Electeur de Leinster, mon adversaire [...] je me flatte que vous ne la publierez pas en son absence ${ }^{22}$." C'est

${ }^{19}$ Cécile Pasteur à Madame Duvernay, 27 mai 1816, $P D$, pièce 8. 20 Loc. cit.

21 La même à la même, 19 janvier 1816, PD, pièce 7 .

22 Xavier Lacombe à M. Duvernay, 4 septembre 1816, PD, pièce 10. 
donc Duvernay qui, en l'absence de Pasteur, doit décider de la matière à imprimer et des annonces à publier, comme le laisse entendre le post-scriptum de la même lettre: "Je prie Mr Duvernay de vouloir bien insérer l'annonce cy dessous d'ans le prochain No du Spectateur ${ }^{23}$." Nul doute que le jeune assistant de 17 ans fait des débuts très prometteurs dans le monde des gazettes, par la grande confiance qu'on lui témoigne.

Cette réussite confirme un souhait de Labadie qui incitait Duvernay à s'engager chez Pasteur en 1815, à l'idée qu'il devienne "un homme de génie de Connaissance et de Lettre par la suite. Car dans cette vocation vous ne pouvez qu'être un homme éclairé" 24. Éclairé, Duvernay l'est-il assez pour aller fonder un journal aux Trois-Rivières en août 1817, à l'âge de 18 ans seulement. Étonnante précocité!

Comment expliquer ce départ? Pourquoi aux Trois-Rivières? Disons tout de suite que l'ascendance paternelle du jeune imprimeur a vécu dans la région immédiate des Trois-Rivières. Des considérations sentimentales ont pu motiver le choix de cette ville. Mais Duvernay aurait-il décidé de fonder un journal parce qu'il se trouvait sans emploi à la suite de la disparition du Spectateur devenu le Spectateur canadien? C'est ce que prétend Benjamin Sulte: "La disparition du Spectateur avait laissé le jeune typographe dans l'embarras, mais l'établissement d'une feuille publiée aux Trois-Rivières sous sa direction le lançait tout à coup, le mettait en évidence et lui offrait maintes occasions de manifester ses talents, car il en avait ${ }^{25}$." $\mathrm{Nul}$ doute quant aux talents! Mais le Spectateur canadien imprimé par Charles-Bernard Pasteur a été publié sans aucune interruption au cours de l'année 1817. Cette suggestion de partir pour les Trois-Rivières lui aurait-elle été faite par un de ses amis influents ? L.-A. Fréchette pense que "suivant les conseils de

23 Loc. cit.

24 L.-G. Labadie à Ludger Duvernay, 1 février 1815, PD, pièce 4. 281.

25 Sulte, Anciens journaux des Trois-Rivières, dans BRH, v. 7, 1901: 
Denis-Benjamin Viger, il va fonder dans la cité de La Violette un journal, la Gazette des Trois-Rivières" ${ }^{26}$.

A la suite d'un stage important de quatre ans dans un grand atelier d'imprimerie à Montréal, Duvernay, confiant en luimême, décide de se lancer activement dans le monde des gazettes, de fonder la première qui ait existé aux Trois-Rivières. Avant 1817, plusieurs journaux de Québec et de Montréal circulaient dans cette ville. Le Courrier de Québec, le Montreal Herald comptaient plusieurs Trifluviens parmi leurs lecteurs. Le Canadien de Québec a même son agent aux Trois-Rivières, M. Pierre Bureau, remplacé par M. F.-X. Boivin. Mais jusqu'à l'arrivée de Ludger, aucun journal n'est imprimé dans cette localité même. Il faut donc reconnaître à ce jeune imprimeur la paternité du journalisme trifluvien.

On ne sait exactement quand Duvernay a quitté l'atelier de Pasteur, probablement au printemps de 1817. Néanmoins, en juin de la même année, l'organisation du nouveau journal semble aller bon train. Le prospectus, en date du 25 juin 1817, est publié dans le Spectateur canadien du 5 juillet: "Propositions pour l'impression d'un journal politique et littéraire, qui sera publié le Mardi de chaque semaine et sera intitulé Gazette des Trois-Rivières" ${ }^{27}$. Mardi, le 12 août 1817, paraît le premier numéro du premier journal imprimé et publié aux Trois-Rivières.

On s'étonnera de voir un imprimeur presque encore adolescent lancer de lui-même un journal. Duvernay vivant aux Trois-Rivières ne bénéficie plus maintenant des délicates attentions de Madame ou de Mademoiselle Pasteur. Peut-il envisager seul de s'occuper à la fois de son travail et de sa subsistance? Un concours féminin lui serait grandement utile. C'est Julie, la seconde fille de la famille, qui vient demeurer à l'imprimerie avec le jeune Ludger. Elle quitte Verchères vers la fin de juillet 1817, non sans causer des regrets chez sa grande amie, Cécile Pasteur: "Je ne puis vous laisser partir sans vous écrire pour vous

${ }^{26}$ L.-A. Fréchette, Ludger Duvernay, fondateur de la Société SaintJean-Baptiste, dans l'Ecrin, 7.

27 Le Spectateur canadien, samedi le 5 juillet 1817: 3, col. 3. 
faire mes adieux, j'espérait que vous viendrez en ville avant de partir j'aurait eu bien du plaisir à vous voir car qui scait à présent quand nous nous reverrons, peut être jamais. Je vous souhaite toute sorte de plaisir et de contentement dans votre nouvel établissement 28 ." Julie ne tarde pas à s'établir dans la localité trifluvienne, car "son sincer amy J Léandre Coursolle" de Verchères lui écrit aux Trois-Rivières le 15 août suivant pour lui faire part de son ennui et de son désir de la rencontrer ${ }^{29}$.

Les premiers numéros de la Gazette des Trois-Rivières attirent les commentaires les plus élogieux. Laissons parler un lecteur de Saint-Sulpice s'adressant à Duvernay: "L'on faits beaucoup d'éloges généralement de ta gazette au surplus il n'y aurait rien d'extraordinaire s'il y avait des mécontents et mécontentes si cela était le cas tu serais le premier parfait des hommes ${ }^{30}$."

Il est difficile d'établir si cet ami de Saint-Sulpice ne serait pas impliqué indirectement dans les premières amours de $\mathrm{Du}-$ vernay avec une demoiselle Nadeau du même village. Un oncle de Ludger, Paul Lamorandière, demeurant au même endroit, félicite son neveu pour l'excellente tenue de son journal et ajoute quelques commentaires: "J'ai été surpris de trouver deux gazettes dans la même enveloppe à mon adresse, mais gonzague m'a dit que c'était destiné pour Mlle Nadeau elle est surprise de ne pas recevoir de lettre de toi ${ }^{31}$." Il semble donc exister des relations quelconques entre les deux, relations qui doivent être rompues pour le plus grand bien de Duvernay, au dire de son oncle: "En ce cas là je te conseille bien de n'en rien faire, Car c'est bien malheureux pour un jeune homme qui semble être né pour pratiquer la vertu de se laisser guider par une passion aveugle dont les suites peuvent être funestes à quiconque ne fait pas de réflescion solide ${ }^{32}$." L'oncle, soucieux du

${ }^{28}$ Cécile Pasteur à Julie Duvernay, à Verchères, (de Montréal), 22 juillet $1817, \mathrm{PD}$, pièce 17 .

29 Léandre Coursolle à Julie Duvernay, 15 août 1817, PD, pièce 14 . 30 L.-G. Nolin à Ludger Duvernay, 23 septembre 1817, PD, pièce 24 . 22-23.

$31 \mathrm{P}$. Lamorandière à Ludger Duvernay, 1 septembre 1817 , PD, pièce

32 Loc. cit. 
bien de son neveu, justifie ces conseils prudents par la situation de la personne concernée: "Ainsi mon cher enfant il faut l'oublier d'autant plus que sa réputation ni celle de sa famille n'est pas respecté je doute pas que tu ne fréquente d'autre société mieux établie ${ }^{33}$." Néanmoins l'oncle Lamorandière ne veut tout de même pas enlever des abonnés au journal trifluvien, lorsqu'il ajoute en post-scriptum: "Si M. Nadeau a souscrit à ton papier, je continuerai de lui faire tenir quand à ce que je viens de citer à leur égard ${ }^{34 . "}$

En juin 1820, Duvernay lance un autre périodique, l'Ami de la Religion et du Roi, journal ecclésiastique, politique et littéraire, qui reproduit des textes d'écrivains religieux français, et aussi des nouvelles purement ecclésiastiques. La publication de ce journal ne semble guère dépasser quelques mois.

Moins de trois ans plus tard, le jeune imprimeur des TroisRivières annonce le lancement d'un troisième journal, le Constitutionnel, dont la présentation ressemble beaucoup à celle de la Gazette des Trois-Rivières. La vie de cette feuille périodique est éphémère, à peine deux ans. Duvernay ne peut rester inactif. Une circonstance politique, l'élection de l'automne 1826 aux Trois-Rivières, inspire à l'imprimeur la mise sur pied d'une autre gazette, l'Argus, pour la durée de la campagne électorale. Nous aurons l'occasion de parler davantage de ces journaux, au cours de notre deuxième chapitre.

De l'atelier de Duvernay ne sortent pas uniquement des journaux. Plusieurs livres, brochures, voient le jour dans l'imprimerie de la rue Royale. En 1819, Duvernay imprime un pamphlet qui se rapporte à la Compagnie du Nord-Ouest. Il en reçoit une commande de S. H. Wilcocke de Montréal: "A l'égard des écrits de M. D. ou d'autre écrivain de la part de Lord Selkirk, le système qu'adopte la Compagnie du Nord-Ouest est de ne jamais commencer aucune attaque, mais aussi de ne jamais laisser aucun écrit sans réponse. Veuillez du reste avoir la

${ }^{33}$ Loc. cit.

34 Loc. cit. 
bonté de m'envoyer une douzaine d'exemplaire du pamphlet que vous êtes occupé à imprimer, quand cela paraît ${ }^{35}$."

On sait que des escarmouches avaient éclaté entre les colons de Lord Selkirk en Assiniboine et les traiteurs de la Compagnie du Nord-Ouest.

Peut-être que la Compagnie, ne voulant "laisser aucun écrit sans réponse" au sujet de ces différends, fait publier une brochure pour bien établir les faits du conflit.

En février 1820, on demande à Duvernay d'imprimer un catéchisme "électoral" pour le compte d'un organisateur de Rivière-du-Loup en Haut: "Imprimer de suite je vous prie le catéchisme cy joins, et n'oublier pas d'envoyer vos gazettes de suite à la Rivière du Loup, Machiche, ou l'élection aura lieu dans huit jours. Je ne veux poins que mon temps sois perdu, surtout dans un ouvrage que je crois utile au public ${ }^{36}$."

L'année 1823 est marquée par des discussions au sujet du gouvernement ecclésiastique du district de Montréal. Les abbés Chaboillez, de Longueuil, et Cadieux, des Trois-Rivières, alimentent une polémique vive qui secoue le monde religieux. Le grand Vicaire du diocèse de Québec publie ses Observations sur un écrit intitulé: Questions sur le gouvernement ecclésiastique du district de Montréal, par un prêtre du diocèse de Québec. La brochure est imprimée par Ludger Duvernay, rue Royale, en $1823^{37}$. Duvernay avait imprimé en 1820 un journal, l'Ami de la Religion et $d u R o i$, qui donnait la première place aux articles d'incidence religieuse.

L'imprimeur des Trois-Rivières se charge aussi de publier des livres pour les écoles. Duvernay imprime en 1824 un Nouveau traité abrégé de la sphère, d'après le système de Copernic.

$35 \mathrm{~S}$. H. Wilcocke à Duvernay, 7 juin $1819, \mathrm{PD}$, pièce $38 \mathrm{a}$.

36 A. G. Douglas à Duvernay, 25 février 1820, PD, pièce 44.

37 Lionel Groulx, Notre maître le passé, III : 189, note 27. Narcisse-Eutrope Dionne, dans son Inventaire chronologique des livres, brochures, journaux et revues, signale la publication de cette brochure qu'il fait remonter par erreur à 1828 . 
Selon Narcisse-Eutrope Dionne ${ }^{38}$, ce manuel scolaire est destiné aux élèves du Séminaire de Québec. Claude Lessard affirme que ce traité est publié à l'intention des élèves du Séminaire de Nicolet ${ }^{39}$. Peut-être que des exemplaires sont spécifiquement imprimés en fonction de chacune des institutions, sans que le contenu général en soit modifié pour cela. Car Duvernay annonce dans le Constitutionnel la nouvelle édition de ce traité “à l'usage des Écoles de cette Province" ${ }^{40}$.

Est-ce que les commandes d'imprimés peuvent suffire à tenir occupés les employés qui travaillent dans l'atelier typographique situé au coin des rues Royale et Plaisante? Le nombre relativement restreint de publications oblige un imprimeur à diversifier davantage son activité. Ainsi, Duvernay annonce dans la Gazette des Trois-Rivières une petite collection de livres de piété tels: Instructions chrétiennes pour les jeunes gens, Histoire abrégée de l'Ancien Testament et de la vie de N.S. J.C., le Petit catéchisme du diocèse de Québec, le Petit livre des enfants, ou quelques manuels, comme le Nouvel alphabet français, l'Arithmétique en quatres parties ${ }^{41}$. Cette double fonction d'imprimeur et de libraire est courante à l'époque.

Et elle n'est pas incompatible avec celle de relieur. Effectivement, en juin 1824, dans une annonce intitulée Reliure, Duvernay

prend la liberté d'informer les dames et messieurs des Trois-Rivières et des environs qu'il a formé des arrangements pour exécuter tout ouvrage dans la branche ci-dessus d'après les nouvelles méthodes anglaises et françaises; il prie en conséquence les personnes qui pourraient avoir des Brochures, des Pamphlets ou des journaux à faire relier, ou de vieux livres à faire réparer, de vouloir bien les envoyer à cette imprimerie, où ils seront réparés avec propreté et élégance. Les ordres de la campa-

38 Dionne, op. cit.: 23.

39 Claude Lessard, Histoire de l'éducation au Séminaire de Nicolet, 1803-1863, 167.

40 Le Constitutionnel, 14 septembre 1824 : 3, col. 1.

41 Gazette des Trois-Rivières, 23 septembre 1817: 1, col. 1. 
gne seront ponctuellement exécutés et les prix seront très modérés ${ }^{42}$.

Les "arrangements" que Duvernay a pris, avec sans doute un autre relieur, laissent entendre qu'il n'effectue pas lui-même le travail, parce qu'il ne dispose pas de l'outillage nécessaire. Car à la fin de l'été 1824, il charge un employé d'un atelier de reliure de Québec de vérifier l'état de quelques outils que Duvernay veut acheter. Le relieur québecois lui rend compte de sa mission le 22 septembre 1824: "A l'égard des outils de M. Lodge, je les ai tout vu et visité après avoir fait attention aux ceux que nous avons, ils ne sont pas meilleurs mais ils sont encore bien bons, pour le prix qu'il vous les a laissé il ne sont payés plus que la moitié de leur juste valeur ${ }^{43}$." Duvernay a pu profiter des conditions avantageuses décrites pour équiper son atelier de machines à relier. Les commandes sont placées en grand nombre, s'il faut en croire un client qui s'impatiente de ne pas recevoir les livres confiés à l'atelier de reliure de Duvernay: "Je vous prie de penser à mes livres, d'envoyer chez mon père ceux qui sont reliés afin que je puisse les rapporter avec moi des 3 Rivières vers le huit de l'autre mois ${ }^{44}$."

Avec l'arrivée de Duvernay aux Trois-Rivières, les gens de cette ville et des environs bénéficient des services d'une imprimerie, d'une librairie, d'un atelier de reliure. C'est une contribution immense qui favorise grandement la vie des "choses de l'esprit'. Cependant le séjour du jeune imprimeur va laisser une empreinte matérielle dans le paysage trifluvien. Albert Tessier, le chantre de la vallée mauricienne, résume très bien le passage de Duvernay dans la cité de Laviolette: "Ludger Duvernay, durant son séjour chez nous, entre 1817 et 1827, semble s'être préoccupé de relever le niveau matériel et intellectuel de sa ville d'adoption. Il fonda des cercles littéraires et des journaux; il procéda aussi à des travaux qui s'inspiraient d'un certain souci d'urbanisme ${ }^{45}$."

\footnotetext{
${ }^{42}$ Le Constitutionnel, 1 juin 1824: 1 .

43 J. B. Tapin à Ludger Duvernay, 22 septembre $1824, \mathrm{PD}$, pièce 55. $\mathrm{PD}$, pièce 61 .

44 L. R. Kimber à Ludger Duvernay, (de Montréal), 29 décembre 1826, 45 Albert Tessier, Trois-Rivières, 1535-1935 (Trois-Rivières, 1935), 145.
} 
Les efforts déployés par Duvernay pour assurer le relèvement du niveau intellectuel de son entourage incitent le jeune homme à remplir une fonction sociale dans son milieu. Ainsi, l'imprimeur de la Gazette des Trois-Rivières est "prié de se trouver à un petit parti qui aura lieu mercredi prochain le 24ième de ce mois à six heures PM" ${ }^{46}$. Duvernay se range parmi les hommes "distingués" qui font l'orgueil des Trois-Rivières. Car cette ville "était fière de posséder alors les abbés $\mathrm{De} \mathrm{Ca}$ lonne, Noiseux et MM. Bédard, Mondelet, Vallières, Duvernay, etc. Naturellement, M. Cadieux se porta vers cette pléiade d'hommes distingués, et les uns les autres devinrent bientôt des amis" ${ }^{47}$. L'imprimeur local s'occupe aussi de recueillir les abonnements pour le Spectateur canadien de Montréal ${ }^{48}$. Ou encore, on retrouve son nom au bas d'une pétition datée du 9 avril 1821 demandant à lord Dalhousie de ne plus utiliser comme prison le vieux monastère des Récollets affecté à cette fin depuis plus de cinquante ans ${ }^{49}$.

Sur le plan matériel, Duvernay a été "mêlé" plus que d'autres à toutes les activités de notre ville, en occupant des fonctions importantes dans l'administration municipale comme par exemple: celle de gérant de la voirie de la ville et d'inspecteur des incendies, ponts et chemins des Trois-Rivières" 50 . On dit que c'est Duvernay qui a entrepris de faire baisser la colline du Platon et d'ouvrir à cet endroit une rue du même nom ${ }^{51}$. En 1823, l'imprimeur du Constitutionnel est nommé membre honoraire de la Société d'agriculture des Trois-Rivières ${ }^{52}$

Duvernay joue un rôle important en tant qu'inspecteur du service des incendies connu sous le nom de Société du Feu, de

\footnotetext{
${ }^{46}$ Invitation adressée à M. Duvernay. Billet signé E. Metote, président, et E. Tapin N.P., 24 novembre $1819, \mathrm{PD}$, pièce 40 .

47 Les Ursulines des Trois-Rivières depuis leur établissement jusqu'à nos jours, II: 402.

48 Par ex. le Spectateur Canadien, 6 novembre 1819: 1, col. 1.

49 Edouard de Saint-Luc, Le fondateur de la Société Saint-Jean-Baptiste, article dans La Presse (date découpée).

50 Henri Vallée, Journaux et journalistes d'autrefois, dans le Nouvelliste, 10 juillet 1948: 11 . Rivières.

51 Renseignements obtenus oralement de M. Hervé Biron des Trois-

52 Le Constitutionnel, 2 septembre 1823: 3, col. 2.
} 
novembre 1819 à la fin de l'année 1826. Il signe par exemple le 29 novembre 1823 une Liste et Inventaire des Effets appartenant à la Société du Feu des Trois-Rivières ${ }^{53}$. Cette fonction lui procure un salaire de six livres par année ${ }^{54}$. Une résolution passée le 27 juin 1827, lors d'une assemblée de cette société, demande "que Noël Pratte soit nommé Inspecteur au lieu et place de Ludger Duvernay et soit substitué en ses droits et privilèges" " 55 . A ce moment, l'imprimeur avait regagné Montréal pour prendre charge de la Minerve.

Lorsqu'il quitte les Trois-Rivières, Duvernay, âgé de 27 ans seulement, laisse une empreinte très marquée dans le sol trifluvien. Son dévouement pour assurer le bien-être intellectuel et matériel de la population ne connaît pas de limites. Il ne lui reste guère de temps pour penser à lui-même. Julie l'a quitté depuis qu'elle s'est mariée le 20 avril $1820^{56}$. Le père de Duvernay se noie en même temps que son gendre, en août 1820, alors que les deux traversent le fleuve Saint-Laurent à la hauteur de Saint-Sulpice ${ }^{57}$. Sa mère, remariée à Joseph Beauchamp en 1822, se sépare de lui en janvier $1824^{58}$. Elle décède en juin de la même année, à la suite d'une longue maladie ${ }^{59}$.

Cependant Duvernay trouve remède à sa solitude. Le 14 février 1825, il se rend à l'église de Saint-Antoine de la Rivièredu-Loup en Haut pour épouser Marie-Reine Harnois, fille majeure d'Augustin Harnois, écuyer de cette paroisse ${ }^{60}$. Informé de cette nouvelle quelque temps auparavant, le député Ranvoysé des Trois-Rivières écrit à Duvernay: "Je vous félicite sur votre choix, vous ne pouvez pas mieux faire. Mais suivant moi votre mariage est un peu prématuré ${ }^{61}$." On peut rester perplexe devant une remarque aussi personnelle d'un homme affecté

${ }_{53}$ Documents relatifs à la Société du Feu, AVTR.

54 Ibid.

55 Ibid.

56 P.-G. Roy, La famille Rocbert de la Morandière, 36.

57 Ibid., 35.

58 Rocbert de la Morandière à ..., 16 janvier 1824, PD, pièce 51.

59 Etienne Gauvreau à ..., 22 juin $1824, \mathrm{PD}$, pièce 54 .

60 Registre paroissial de Saint-Antoine de la Rivière-du-Loup. Acte rapporté par Parent, Duvernay le Magnifique, 10.

61 Etienne Ranvoysé à Ludger Duvernay, 9 février 1825, $\mathrm{PD}$, pièce 57. 
à la fonction publique. Duvernay aurait-il annoncé son mariage d'une façon inopinée ? Ou existe-t-il une cause qui peut précipiter l'échange du consentement mutuel ? Il faut mentionner que les deux parties ont "obtenu dispense de deux bans par lettre de Messieu Noiseu, grand vicaire, en date du douze du présent mois" ${ }^{62}$, soit deux jours seulement avant la célébration de la cérémonie nuptiale. Le député termine sa lettre en disant: "Dans le cas que vous persistier à vous nupter je vous souhaite prospérité ${ }^{63}$." Ce souhait est bientôt comblé. Le 30 octobre de la même année naît Julie-Hortense Duvernay, fille de l'imprimeur trifluvien ${ }^{64}$.

Le départ assez soudain de Duvernay pour Montréal surprend quelque peu, étant donné l'ampleur et le succès du travail fourni durant une décennie. On prie Duvernay de se charger de la direction de la Minerve suspendue après la publication de quelques numéros, en novembre 1826. Mais au cours de son séjour aux Trois-Rivières, l'imprimeur local a reçu plusieurs offres qui lui demandaient d'exercer sa profession à Montréal.

J. R. Kimber, qui s'occupe du Canadian Spectator à Montréal, fait à Duvernay la proposition suivante:

Je suis enfin autorisé à vous demander si vous êtes dans la disposition de nous vendre votre Presse et votre imprimerie, votre plus bas prix, ainsi que les termes de payement ${ }^{65}$.

Kimber veut bel et bien acheter les presses, non pas confier l'impression de son journal à Duvernay:

je pourrais vous garantir la préférence comme imprimeur car nous sommes décidés à acheter une imprimerie et non à faire imprimer notre papier, comme nous le fesions ci-devant ${ }^{66}$.

62 Registre paroissial ..., loc. cit.

63 Etienne Ranvoysé à Ludger Duvernay, 9 février 1825, loc. cit.

64 P.-G. Roy, op. cit., 37.

65 J. R. Kimber à Ludger Duvernay, (de Montréal), 11 février 1824, $\mathrm{PD}$, pièce 52.

66 Loc. cit. 
Duvernay refuse sans doute cette offre. Car, moins de deux semaines après la lettre de Kimber, c'est Jocelyn Waller du même journal qui lui fait part de nouvelles conditions: "I beg to know from you if you would have any objection to transfer your Press to this town immediately and print my paper ${ }^{67}$." Duvernay se voit offrir la charge d'imprimer le Canadian Spectator. Mais Waller lui fait part en même temps d'une suggestion encore plus alléchante: "I apprehend that an opportunity of a most excellent kind is now open to establish a good french Paper either separately or in the same sheet with English ${ }^{68}$." Voilà une proposition qui ne manque pas d'intérêt pour l'imprimeur de 25 ans. Peut-être Duvernay ne se sent-il pas suffisamment rodé au journalisme pour réaliser cette ambition. Mais la nouvelle offre que lui fait Kimber, à la fin de l'année 1826, contient une proposition vraiment intéressante: "La vacance d'imprimeur du Canadian Spectator se présente, depuis le départ de M. Jones pour les États-Unis. Je vous ai proposé pour la remplir, à la dernière assemblée du Comité, on m'a chargé de vous en écrire aussitôt et de vous demander si vous accepterier la charge de tout l'établissement et de le conduire à votre profit et dépens ainsi qu'il l'était par le ci devant imprimeur 69." Ayant la charge de "tout l'établissement", Duvernay pourra imprimer le Canadian Spectator de Waller, et aussi un journal français qu'il dirigera lui-même. Au moment de cette lettre, la publication de la Minerve est suspendue. C'est l'occasion de la relancer. Les décisions sont prises avec rapidité. Si bien que Duvernay imprime le premier numéro de la Minerve le lundi, 12 février 1827, à son atelier situé à 5, rue SaintJean-Baptiste, à Montréal. Jocelyn Waller continue de rédiger son Canadian Spectator qu'il fait imprimer par son nouvel associé. Ce concours de circonstances hâte ainsi le départ de l'imprimeur trifluvien vers Montréal.

67 Jocelyn Waller à Duvernay, (de Montréal), 24 février 1824, Pd, pièce 53.

68 Loc. cit.

${ }^{69}$ L. R. Kimber à Ludger Duvernay, (de Montréal), 29 décembre 1826, $\mathrm{PD}$, pièce 61 . 
$\mathrm{Au}$ cours de ces dix années passées aux Trois-Rivières, Ludger Duvernay laisse une trace remarquable au pays de ses ancêtres. Imprimeur, libraire, relieur, inspecteur des incendies, officier de voirie, membre de sociétés diverses, ce ne sont que quelques tâches dans lesquelles se cristallise l'énergie débordante de celui qui deviendra "l'un des plus remarquables journalistes que l'Amérique du Nord ait jamais produits" ${ }^{70}$.

(à suivre)

Yves Tessier, Bachelier ès arts, bachelier en histoire.

70 Wilfrid Bovey, Les Canadiens-Français, d'aujourd'hui, l'essor d'un peuple. Traduit de l'anglais par J.-J. Lefebvre, (Montréal, 1940), 120. 